\title{
Interstitial Pneumonitis Due to Janus Kinase Inhibitor Baricitinib in a Rheumatic Arthritis Patient
}

\author{
Yalda Hadizamani ${ }^{1,2, \$}$, Fabian Messmer ${ }^{1,2, \$}$, Lia Bally ${ }^{5,2}$, Arthur Helbling ${ }^{6}$, Thomas Lehmann ${ }^{3, \#}$, and Juerg \\ Hamacher ${ }^{1,2,7, \#, *}$
}

\$,\#: Both authors equally contributed to the manuscript.

${ }^{1}$ Internal Medicine and Pneumology, Lindenhofspital, Lindenhofgruppe Bern, Switzerland

${ }^{2}$ Lungen- und Atmungsstiftung Bern, Switzerland

${ }^{3}$ Surgery for Rheumatology and Osteology, Osteo Rheuma Bern AG, Switzerland

${ }^{4}$ Internal Medicine, Sonnenhofspital, Switzerland

${ }^{5}$ Department of Endocrinology and Diabetology, Inselspital, Bern, Switzerland

${ }^{6}$ Department of Rheumatology, Immunology and Allergology, Inselspital Bern University Hospital, University of Bern, Switzerland

${ }^{7}$ Department of Internal Medicine V - Pulmonology, Saarland University Hospital, Germany

Submission: September 23, 2020; Published: October 14, 2020

*Corresponding author: Juerg Hamacher M.D, Lindenhofspital Bern, Bremgartenstrasse 119, CH-3012 Bern, Switzerland,

Email: hamacher@greenmail.ch

\section{Abstract}

A 68 years old rheumatoid arthritis patient received the janus kinase inhibitor baricitinib after a 2.5 years treatment with abatacept. Under abatacept she developed important cough, which was the reason to directly change to baricitinib. Within days cough was much aggravated and baricitinib treatment stopped after two weeks. The cough decreased, and six-week later baricitinib treatment was restarted. Within 14 days cough reappeared and novel exertional dyspnoea and hypoxaemia, aggravated with low exercise, and erythema at the face, neck, upper trunk, volar arms including wrists developed.

Clinical findings, a novel restrictive lung function and gas diffusion impairment, and computer tomography were consistent with an acute pneumonitis due to baricitinib treatment. Baricitinib was stopped, and recovery was virtually complete within six weeks. We conclude that a very probable baricitinib pneumonitis occurred. To our knowledge, this is the first published case of this adverse effect in a rheumatoid arthritis patient.

Keywords: Baricitinib; Autoimmune diseases; Cytokines; Pneumonitis; Gastroesophageal

Abbreviations: JAK: Janus Kinase; RA: Rheumatoid Arthritis; Anti-CCP: Anti-Cyclic Citrullinated Peptide; ANA: Antinuclear Antibodies; TYK2: Tyrosine Kinase 2; tsDMARD: Targeted Synthetic Disease Modifying Anti Rheumatic Drug; ILD: Interstitial Lung Disease

\section{Introduction}

Baricitinib is one of the first small molecules, the oral Janus Kinase (JAK) inhibitors and has been approved in 2017 by the European Medical Agency for the treatment of Rheumatoid Arthritis (RA). By intracellularly inhibiting the four janus kinases in humans, it is highly selective towards essential downstream mediators of many clinically central pro-inflammatory cytokines, the major drivers of autoimmune diseases like RA [1]. It mainly inhibits JAK1 and 2, has moderate activity against TYK2 and an about 100 fold less activity against JAK3 inhibition compared to that of JAK 1 and 2 [2,3].

Baricitinib has anti-inflammatory and antiviral properties and may improve the outcome of coronavirus disease 2019 (COVID-19) patients [4,5], targeting immune dysregulation triggered by inhibition of cytokine release [6,7] and by reducing the virus ability to enter lung cells [6]. Combining baricitinib with other anti-inflammatory or with antiviral drugs may thus decrease 
viral infectivity and replication as well as exaggerated tissuedestructive inflammatory response such as acute respiratory distress syndrome [5].

According to huge phase III clinical trials [8] baricitinib is described to usually lead to rapid improvements in disease activity, functional and patient reported outcomes, in part already observable within one week. It appears to have become an important pillar in RA therapy. We report on a female rheumatoid arthritis patient that stopped baricitinib intake due to severe cough aggravation and suffered from a very probable baricitinib pneumonitis during a re-exposition of the drug two weeks later.

\section{Case Description}

A 68 years old female with a 38 years history of an erosive form of rheumatoid arthritis with positive rheuma factors, AntiCyclic Citrullinated Peptide (Anti-CCP) antibodies and Antinuclear Antibodies (ANA) was treated with baricitinib after a 3 years period of treatment with golimumab and changed to abatacept due to possibly associated nausea and hair loss. During 2.5 years abatacept therapy she developed important cough, which was the reason to directly change to baricitinib. However, within days cough without phlegm was much aggravated under the treatment with baricitinib.
The patient therefore stopped baricitinib intake after two weeks of treatment for a two-month period. In the following period the cough was reduced; a possible gastroesophageal reflux component was further treated with conservative measures, omeprazole, regular sodium alginate, and oral magnesium hydroxide and aluminium hydroxide $\left(\right.$ Alucol $\left.^{\mathrm{R}}\right)$ as needed. Due to important joint and periarticular pain, baricitinib was re-started two month after its stop, which led to a novel aggravation of cough and a novel exertional dyspnoea, hypoxaemia with severe aggravation at low exercise, and a minimal erythema with a squamous component at the face, neck, upper trunk, volar arms including wrists within one to two weeks. Lung auscultation revealed very few end inspiratory crackles at both lung bases.

Respiratory rate was at about 22/', heart rate 105/'. Pulmonary function tests showed a moderately severe restrictive pattern of $25 \%$ less (vital capacity, FEV1, CO diffusion capacity) than normal values two months before. Due to severity and the possibility of pulmonary embolism, ventilation perfusion lung scintigraphy ruled out this differential diagnosis. Computer tomography, normal 2 months before, confirmed an interstitial pneumonitis with ground glass infiltrates and dense infiltrates. At this time, 15 days of this second period of treatment, baricitinib was stopped (Table 1).

Table 1:Evolution of lung functional data during Pneumonitis.

\begin{tabular}{|c|c|c|c|c|c|}
\hline Days Since Treatment Onset & -45 & 15 & 31 & 82 & 149 \\
\hline $\mathrm{VC}[\mathrm{L}]$ & 2.43 & 1.42 & 1.81 & 2.17 & 2.18 \\
\hline$\%$ Predicted & 92 & 54 & 69 & 82 & 83 \\
\hline FEV1 [L] & 1.73 & 1.13 & 1.44 & 1.78 & 1.80 \\
\hline$\%$ Predicted & 78 & 51 & 65 & 80 & 81 \\
\hline DLCO & 6.8 & 5.2 & 5.0 & 6.4 & 6.3 \\
\hline$\%$ Predicted & 91 & 70 & 67 & 86 & 85 \\
\hline $\mathrm{O}_{2}$ At rest $\%$ & $95-96$ & $90-92$ & $95-96$ & $95-96$ & $95-96$ \\
\hline $\mathrm{O}_{2}$ Exercice \% & 92 & 87 & 91 & 92 & 92 \\
\hline
\end{tabular}

Blood tests showed a normal white cell count of $4.6 \times 10^{9} / \mathrm{l}$ and platelet count and moderate lymphocytopenia of $0.6 \times 10^{9} / \mathrm{l}$, a C- reactive protein of $50 \mathrm{mg} / \mathrm{l}(\mathrm{N}<5)$, a normal procalcitonin of $0.08 \mu \mathrm{g} / \mathrm{l}(\mathrm{N}<0.1) ; \mathrm{LDH}$, fibrinogen and $\mathrm{D}$-dimer test were at about $1.5 \mathrm{x}$ upper normal value; tryptase and IgE were normal. The associated aggravated cough at the first 2 weeks of exposition re-aggravated within days of the re-exposition with baricitinib and showed a rather rapid clinical recovery of dyspnea, cough and rush after stopping the second exposition of the drug. According to this course of treatment and symptoms and the missing clinical and paraclinical indication for alternative diagnoses we diagnosed a baricitinib induced interstitial pneumonitis, accompanied by a mild skin rash.

(Figure 1) Very moderate ground glass pattern and mainly basal, chiefly subpleural denser consolidations and not clearly limited nodular infiltrates and moderately thickened bronchial walls during acute pneumonitis attributed to baricitinib. Note that a CT scan showed normal pulmonary structures two month before.

\section{Discussion}

Baricitinib is a small molecule targeted synthetic Disease Modifying Antirheumatic Drug (tsDMARD) selective towards intracellular JAK1 and 2 inhibition, has moderate activity against the janus kinase member Tyrosine Kinase 2 (TYK2) and much less activity against JAK3 inhibition. It exhibits specifity for JAK1 and JAK2 with IC50 values in the nanomolar range $[2,8]$.

Known side effects of JAK inhibitors can frequently be linked to the action of the cytokines that are blocked [1]. Thus, increased viral infections as herpes zoster or upper respiratory tract 
infections are observed, and, similar to the disease-modifying anti-rheumatic biologicals, infections including tuberculosis or osteomyelitis. Rather rarely they lead to anaemia or cytopenia, cause nausea, increase LDL cholesterol, or thrombocytosis [3,810].

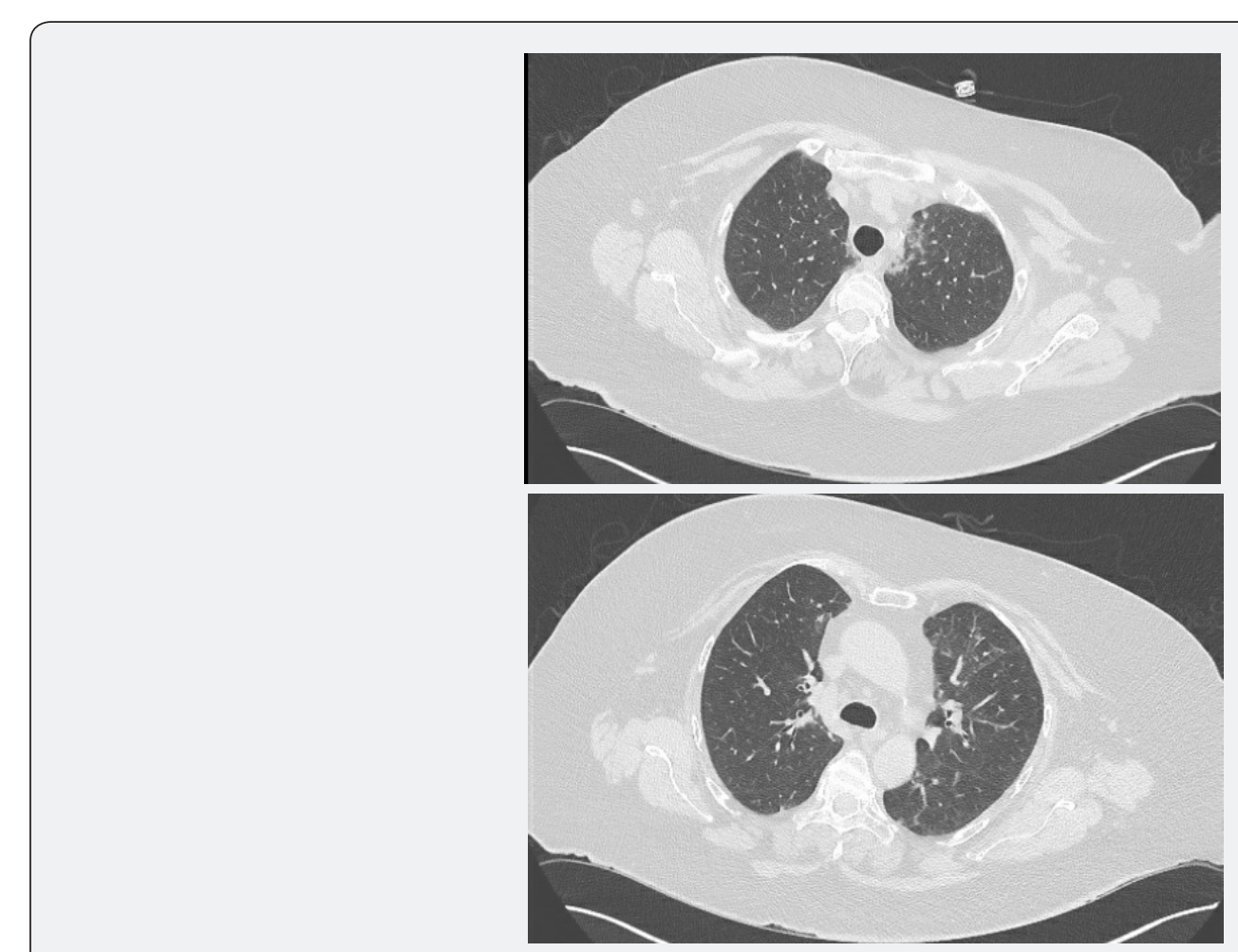

Figure 1: Very moderate ground glass pattern and mainly basal, chiefly subpleural denser consolidations and not clearly limited nodular infiltrates and moderately thickened bronchial walls during acute pneumonitis attributed to baricitinib. Note that a CT scan showed normal pulmonary structures two month before.

As the JAK inhibitors are relatively new in the treatment portfolio, data on safety for rarer adverse events are only slowly accumulating, and novel inhibitors will probably enter the clinical world. So far, there is no case of pulmonary adverse event published with baricitinib according to the best of our knowledge. However, with tofacitinib in RA treatment, Harigai [6] discussed a total of 14 persons with serious interstitial lung disease and described that tofacitinib patients developed in $0.1 \%$ Interstitial Lung Disease (ILD) and some of them as de novo interstitial lung disease.

According to Harigai [6], a Japanese interim post-marketing analysis has identified 14 cases $(0.5 \%)$ with serious ILD, of which three died. The most recent analysis of 21 published clinical trials covering 7061 patients and 23394 patient years of treatment showed similar results with 42 patients being probably or possibly affected, being $0.6 \%$ or 0.18 per 100 patient-years, with $83 \%$ classified as mild to moderate, but 3 of the 42 leading to death [7]. This analysis only included very few patients of the work of Harigari [6]. Furthermore, one case of pulmonary hypertension has been described under tofacitinib [11].

Interstitial lung disease can occur in rheumatoid arthritis patients and may be important in terms of outcome like quality of life, disability, or even mortality. Disease modifying drugs involved in rheumatoid arthritis treatment may themselves cause druginduced pneumonitis, but could on the other hand theoretically also inhibit this type of specific disease manifestation of rheumatoid arthritis, which occurs about in a frequency of 0.2 events per 100 patient years [12].

We add therefore, this case report as a first publication on baricitinib, underlining that severe interstitial pneumonitis could be an untoward effect of the drug. The virtually missing clinical hints towards any viral infection and the clear course of exposure and symptoms with rather acute onset of cough, exercise intolerance and severe gas exchange, the improvement of exercise tolerance and cough after stopping the treatment, and as well a huge recovery one month and the complete recovery three months later, foster the diagnosis of drug-induced pneumonitis due to baricitinib. Whether such adverse pulmonary event is due to direct cytokine effect modifications under baricitinib or is due to hypersensitivity remains open.

Accordingly, a limitation of our report is that we cannot completely exclude an infective origin, especially not a viral one. The strong exposition and re-exposition time association with important symptoms however make the hypothesis of an infective 
or other origin highly unlikely.

\section{Acknowledgement}

The work was supported from the Lungen- und Atmungsstiftung Bern to Yalda Hadizamani. The work was further supported by a grant from the Stiftung Lindenhof Bern (13-09-F) to Juerg Hamacher.

\section{References}

1. Schwartz DM, Bonelli M, Gadina M, JJ O'Shea (2016) Type I/II cytokines, JAKs, and new strategies for treating autoimmune diseases. Nat Rev Rheumatol 12(1): 25-36.

2. Clark JD, Flanagan ME, Telliez JB (2014) Discovery and development of Janus kinase (JAK) inhibitors for inflammatory diseases. J Med Chem 57(12): 5023-5038.

3. Schwartz DM, Kanno Y, Villarino A, Ward M, Gadina M, et al. (2017) JAK inhibition as a therapeutic strategy for immune and inflammatory diseases. Nat Rev Drug Discov 17(1): 78.

4. Lo Caputo S, Corso G, Clerici M, Santantonio TA (2020) Baricitinib: A chance to treat covid-19? J Med Virol.

5. Stebbing J, Phelan A, Griffin I, Tucker C, Oechsle O, et al. (2020) Covid-19: Combining antiviral and anti-inflammatory treatments. The Lancet Infectious diseases 20(4): 400-402.
6. Richardson P, Griffin I, Tucker C, Smith D, Oechsle O, et al. (2020) Baricitinib as potential treatment for 2019-ncov acute respiratory disease. Lancet 395 (10223): e30-e31.

7. Cantini F, Goletti D, Petrone L, Najafi Fard S, Niccoli L, et al. (2020) Immune therapy, or antiviral therapy, or both for covid-19: A systematic review. Drugs.

8. Taylor PC (2019) Clinical efficacy of launched JAK inhibitors in rheumatoid arthritis. Rheumatology (Oxford) 58(Supplement-1): i17-i26.

9. Al Salama ZT, Scott L J (2018) Baricitinib: A Review in Rheumatoid Arthritis. Drugs 78(7): 761-772.

10. Harigai M (2019) Growing evidence of the safety of JAK inhibitors in patients with rheumatoid arthritis. Rheumatology (Oxford) 58(Supplement-1): i34-i42.

11. Habib P, Podgorski M, Adorini K, Brown M, Mc Lean A, et al. (2018) First Reported Case of Pulmonary Arterial Hypertension Secondary to Tofacitinib Treatment for Undifferentiated Arthritis. J Clin Rheumatol.

12. Citera G, Mysler E, Madariaga H, Cardiel MH, Castaneda O, et al. (2020) Incidence Rates of Interstitial Lung Disease Events in TofacitinibTreated Rheumatoid Arthritis Patients: Post Hoc Analysis From 21 Clinical Trials. J Clin Rheumatol.

Your next submission with Juniper Publishers will reach you the below assets

- Quality Editorial service

- Swift Peer Review

- Reprints availability

- E-prints Service

- Manuscript Podcast for convenient understanding

- Global attainment for your research

- Manuscript accessibility in different formats ( Pdf, E-pub, Full Text, Audio)

- Unceasing customer service

Track the below URL for one-step submission https://juniperpublishers.com/online-submission.php 